\title{
Benchmarking the Robustness of Cellular Up-Links in Automatic Weather Station Networks
}

\author{
Emmanuel A. Kondela1,2, Amos Nungu'2, Joseph W. Matiko², Julianne S. Otim¹, Bjorn Pehrson ${ }^{3}$ \\ ${ }^{1}$ School of Computing and Information Technology, Makerere University, Kampala, Uganda \\ ${ }^{2}$ Department of Computer Studies, Dar es Salaam Institute of Technology, Dar es Salaam, Tanzania \\ ${ }^{3}$ Telecommunication Systems Lab, Royal Institute of Technology, Kampala, Sweden \\ Email: ekkondela@hotmail.com, sansa@cit.ac.ug, amosnungu@dit.ac.tz, jwmatiko@gmail.com, bpehrson@kth.se
}

How to cite this paper: Kondela, E.A., Nungu, A., Matiko, J.W., Otim, J.S. and Pehrson, B. (2018) Benchmarking the Robustness of Cellular Up-Links in Automatic Weather Station Networks. Communications and Network, 10, 78-92.

https://doi.org/10.4236/cn.2018.103007

Received: November 6, 2017

Accepted: July 27, 2018

Published: July 30, 2018

Copyright $\odot 2018$ by authors and Scientific Research Publishing Inc. This work is licensed under the Creative Commons Attribution-NonCommercial International License (CC BY-NC 4.0). http://creativecommons.org/licenses/by-nc/4.0/

\section{Open Access}

\begin{abstract}
We present a problem for benchmarking the robustness of cellular up-links, in an automatic weather station (AWS) testbed. Based on the problem, we conduct a small-scale measurement study of robustness, where the AWS is equipped with four (4) cellular modems for weather data delivery. The effectiveness of up-links is challenging because of overlapping spatial-temporal factors such as the presence of good reflectors that lead to multi-path effects, interference, network load or other reasons. We argue that, there is a strong need for independent assessments of their robustness, to perform end-to-end network measurement. However, it is yet difficult to go from a particular measurement to an assessment of the entire network. We extensively measure the variability of Radio Signal Strength (RSSI) as link metric on the cellular modems. The RSSI is one of the important link metrics that can determine the robustness of received RF signals, and explore how they differed from one another at a particular location and instant time. We also apply the statistical analysis that quantifies the level of stability by considering the robustness, referring short-term variation, and determines good up-link in comparison to weak one. The results show that the robustness of cellular up-links exists for an unpredictable period of time and lower than one could hope. More than $50 \%$ of up-links are intermittent. Therefore, we plan to extend our work by exploring RSSI thresholds, to develop a classification scheme supporting a decision whether a link is either intermittent or not. This will help in normalizing the level of stability, to design the RSSI estimation metric for the robust routing protocol in weather data networks.
\end{abstract}

\section{Keywords}

Cellular Links, Robustness, Automatic Weather Station, Terrestrial Wireless 
Links, Intermittent Links

\section{Introduction}

Terrestrial wireless communication networks encompass the use of cellular networks or dedicated wireless networks to pass information between terminals. For instance, AWS networks [1] [2] [3], use cellular terminals to enable connectivity with nearby base stations and transmit weather data to central repositories. In literature, much research has explored the stability of cellular up-links on indoor and outdoor environments [4] [5] [6] [7]. In this paper, we concentrate on an indoor environment, and focus on hardware link metric RSSI, to determine the robustness of up-links by considering the level of stability. Its spatial and temporal variations are the main issue of the RSSI variation.

Up-link refers to a one-way interconnecting between a modem and base station for the purpose of transmitting weather data to the central repository. Cellular up-link is dynamic, with the quality of which can change considerably with time and distance, due to the presence of various factors such as the presence of good reflectors (e.g. metal, walls, woods and glass) that lead to multi-path (reflections, fading, diffraction) effects, interference, network load or other reasons. Hardware miss-calibration and antenna position are other two additional factors that may affect signal propagation [8] [9] [10]. Therefore, the mentioned factors may lead to the variation of RSSI with values between $-51 \mathrm{dBm}$ (good) to -113 $\mathrm{dBm}$ (bad) as a result the up-link is intermittent.

Intermittent links sometimes exist as perfect links and sometimes don't exist for an unpredictable period of time. When the topology of the network is at least predictable, then the behaviors of intermittent links can be analyzed. So, in order to understand the robustness of up-link, is necessary to exploit its stability, and to provide meaningful information about its surrounding. The study of robustness of up-links [11], is focused on describing how link metric is affected by surrounding activities. The underlying assumption is that the networks having intermittent links with low RSSI or malfunctioning of some nodes cause the removal of their links at particular time, and thus some paths completely disappear, resulting in the loss of connectivity.

This paper, attempts to answer the following key question, "whether RSSI is suitable metric to determine what level of robustness is expected?" We aim to benchmark the variability of RSSI on cellular up-links, to determine its robustness at a particular location and instant of time. In order to tackle this objective, the paper investigates the spatial and temporal effects on the RSSI, a measure of the radio frequency $(\mathrm{RF})$ signals a power level that a node is receiving. Finally, we focus on understanding what robustness eventually reaches, a steady minimum value by considering the level of stability, whether is good, intermediate or bad. Thus, our paper consolidates the significance of considering RSSI as a link metric, and explores its accuracy to estimate the robustness against spa- 
tial-temporal factors, referring short-term variation. This will help in normalizing the level of robustness, to design RSSI estimation metric for the robust up-link routing protocol in weather data networks. The stability has to avoid long-term variation so that the routing does not have to reconsider alternative up-link, which is energy consuming.

The paper is organized as follows. Section II describes a typical scenario of other related works. Section III presents the experimental setup. Section IV describes some experimental results and analysis. Section V draws conclusions and future work.

\section{Related Work}

In this section, we review related work, including efforts made and challenges faced. In addition, we highlight the gaps that have influenced our work. The works in [12]-[18], use cellular up-link(s) for efficient transmission of weather data. These works highlighted the challenges of intermittent links, and point out a need of assessing the robustness of cellular links, for efficient transmission of data.

There are several methodologies [19] [20] to benchmark the performance impact of cellular network links. They use analytical and numerical evaluation to describe a measure of network robustness for time-varying networks, and showed how they perform on different classes of random models. However, the robustness assessment in cellular networks, introduced a unique set of technical challenges because of service performance on internal and external factors. The performance of intermittent link is influenced by many factors. The impact of these factors can over-shadow the performance assessment in cellular networks. Thus, it is important to carefully account for the both factors in order to make accurate inferences on the impact of up-links that can be expected for better planning of an AWS network.

Other studies of the robustness of networks, have mainly focused on describing how a given performance metric of the network is affected when terminals or nodes are removed [19]. The underlying assumption is that, the absence or malfunctioning of some nodes will cause the removal of their edges and, thus some paths will become longer, increasing the distances between the remaining nodes or completely disappear resulting in the loss of connectivity in a particular link. Therefore, we benchmark and explore the variability of RSSI as link metric to quantify their performances, and evaluate their effectiveness by considering the robustness against networks failures in the presence of internal factors.

\section{Experimental Setup}

The experimental setup is based on AWS testbed, equipped with wireless sensor network (WSN). It is a networked system of interconnected nodes communicating wirelessly and reporting their measurement data to a central repository. In more detail, each such sensor network node has typically several parts: a radio transceiver with an internal antenna, a microcontroller (MCU) an Atmel ATMega128RFA1 [20], an electronic circuit for interfacing with the sensors and an energy source. The topology of the WSNs can vary from a simple star network to 
an advanced multi-hop wireless mesh network. The propagation technique between the hops of the network can be routing or flooding [21]. A WSN is built of four (4) nodes, where each node is connected to one (or several) sensors. For example, 1) a sensor node at $10 \mathrm{~m}$ level collects wind direction, wind speed, and solar radiation. 2) A sensor node at $2 \mathrm{~m}$ level collects atmospheric temperature and relative humidity. 3) A sensor node on the ground, collects soil moisture, soil temperature, and precipitation. 4) A sink node at the gateway, collects atmospheric pressure and reports from the rest of nodes.

\subsection{Sensor Node}

In Figure 1, a node automatically becomes a sink node when connected via a TTL/USB converter to a USB port of a gateway. Each node has the built-in printed circuit board (PCB) antenna, with radio coverage about $300 \mathrm{~m}$ at the line-of-sight (LOS), following the $2.4 \mathrm{GHz}$ IEEE 802.15.4 standard protocol [22]. This is a low power communication option which usually allows 10 - 20 meters of range with an average $250 \mathrm{kbit} / \mathrm{s}$ raw data rate. In our case, the nodes wake up according to a schedule, broadcast messages with sensor data that can be captured by the sinknode and goes back to deep sleep. On top of the IEEE 802.15.4 link protocol, the Contiki's Rime protocol is used to broadcast [23]. During the process of transmission, the collected packets may be handled by multiple nodes to get to a sink node, which is connected next to the gateway. Finally, packets are reaching a repository through the INTERNET using different cellular up-links.
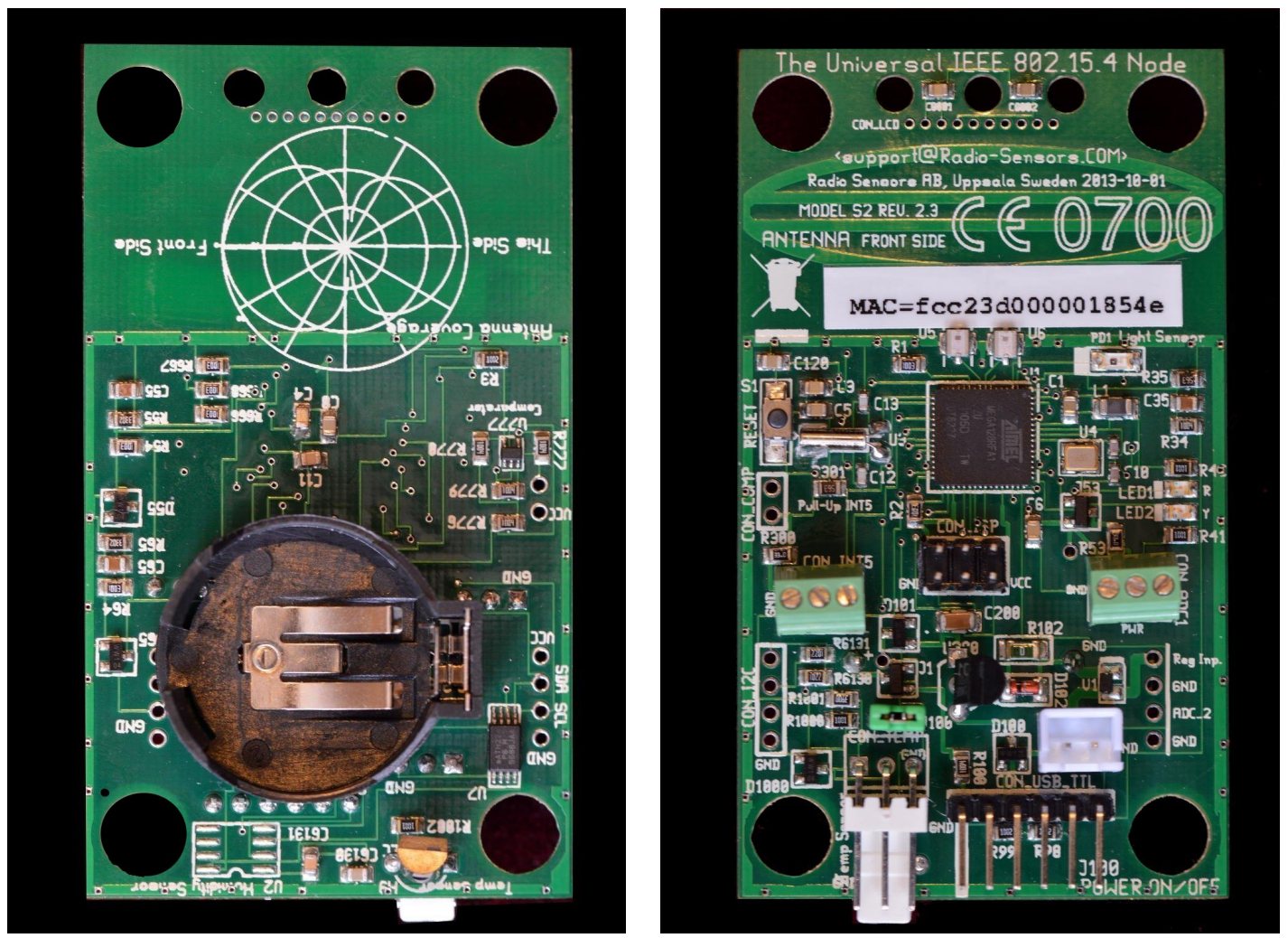

Figure 1. A Radio Sensor (RS) node. 
The AWS unit is powered from at least two different sources: the grid-power and an embedded form of energy harvesting (e.g. solar panels). The gateway and sink node are powered by grid source, the remaining nodes are powered by a 15 W solar panel and ultra-capacitor consisting of two Lithium Ion Capacitor (LIC) via LM2596S step-down converters (regulators). The PSU regulator LM2596S converts any input voltage between $4-24 \mathrm{~V}$ to a constant voltage $(3.6 \mathrm{~V})$ required to operate.

\subsection{Experimental Environment}

The experimental environment is on the $2^{\text {nd }}$ floor in a four (4) floors building, consisting of various rooms. The environmental setup is a high ceilinged building, constructed from concrete blocks (granite). It has thick concrete walls (0.25 $\mathrm{m}$ thick outer walls, and $0.15 \mathrm{~m}$ thick walls between rooms), $5 \mathrm{~m}$ high ceilings, heavy wooden doors, tables and other office equipment. During measurements, the gateway is positioned at three different locations (L1, L2, L3). The distance between L1 - L2 is about $4 \mathrm{~m}$, the distance between L2 - L3 is about $3 \mathrm{~m}$ and the distance between L1 - L3 is about $6 \mathrm{~m}$. Owing to heavy attenuation by walls and doors, link outage in the environment is possible large.

\subsection{Data Acquisition}

The RSSI measurements are conducted by connecting four (4) cellular modems (SIM800L, HSDPA HUAWEI E153, HSDPA HUAWEI E173 and RoHS HSPA ZTE MF665C) at the gateway via standard USB hub 2.0. The modems make connections to the INTERNET from different mobile operators including Halotel, Tigo, Airtel, and Vodacom. During measurements, the experiment is repeated at each location, and RSSI is recorded in real time. The experiment is conducted with an average time of one hour, and the total amount of time it took for a modem to respond to a request for RSSI value is a second (it is the sum of service time and wait time). An open question "How many readings are needed for analyzing a robust link?", remains unanswered. However, various studies claimed to attain a reliable analysis of large historical data above 50 packets [24] [25] [26]. The sampling rate is approximately 1200 samples/hour, thus recorded 7200 samples over 6 hours. Afterwards, the statistical techniques such as minimum, maximum, range, mean, median, variance, standard deviation and coefficient of variation are applied, to estimate the level of stability by comparing the robustness of up-links, referring short-term variation.

\section{Experimental Results and Discussion}

When benchmarking the robustness of cellular up-link, we need to find ways to measure the network performance and determine when links are intermittent, as each network is different in nature and design. Whatever the approach we take to the problem, we use RSSI as link metric that reflects the performance status of the up-link. Therefore, in this section, we present the results regarding our measurements, and analyze the variability of RSSI, and discuss the statistical 
properties of the measured data, and finally benchmark the level of stability by considering the robustness of up-link, referring short-term variation.

\subsection{Variability of RSSI}

The RSSI varies as a function of time and distance. To analyze the variability of RSSI, we consider spatial and temporal variations between receiver and transmitter. Several statistical numerical measures for describing the variation, or spread, or dispersion, of RSSI, are considered including, minimum, maximum, median, range, mean, variance, standard deviation, and coefficient of variation.

Spatial variation: RSSI values vary as a function of a distance between transmitter and receiver. In free space, RSSI is inversely proportional to the squared distance between the transmitter and the receiver.

$$
P_{r}(d)=C_{f}\left(P_{t} / d^{2}\right)
$$

where $P_{r}$ is the received power, $C_{f}$ constant depending on a transceiver, $P_{t}$ transmitting power and $d$ distance. Figure 2 analyzes the spatial properties of RSSI recorded at three different locations L1, L2 and L3. Hence, the base stations are observed at both locations and the levels of RSSI are compared. The strength of RSSI at L2, is more weaker than L1, and at L3 is much weaker than L2 and L1. This is due to the fact that, multi-path fading is the most common factor that affects RSSI variation resulting in a large deviation from the path loss models. The propagation of radio signal attenuates with the increase of the distance, which may be affected by reflection, diffraction, and scattering. For instance, reflection is one of the main factors of multi-path presence that may alter the link reliability through huge drops of the packet reception ratio, when a propagating radio signal impinges upon surfaces or objects. This occurs due to the presence of good reflectors such as concrete walls, wooden, metal and glass, and leads not just to the multi-path effect, but also to the noise increase and interference. These structures weaken or completely prevent radio signals leading to RSSI variations. Therefore, reflection, scattering, and other physical properties have an extreme impact on RSSI measurement, especially in indoor environments.

Tables 1-3 indicate the statistical data for the consistent four (4) mobile operators at locations L1, L2, and L3. For instance, the range difference at two locations $\mathrm{L} 1$ and $\mathrm{L} 2$ is $2 \mathrm{dBm}$ for Halotel, $32 \mathrm{dBm}$ for Tigo, $-4 \mathrm{dBm}$ for Vodacom and $11.3 \mathrm{dBm}$ for Airtel. In the second experiment, the range difference between two locations L1 and L2 is $23 \mathrm{dBm}$ for Halotel, $6 \mathrm{dBm}$ for Tigo, $-2 \mathrm{dBm}$ for Vodacom and $5.7 \mathrm{dBm}$ for Airtel. This means, ranges of RSSI are not constant, but this does not tell us how the RSSI observations are distributed between the smallest and the largest ones. The only information we have from the range is the distance between the smallest and the largest measurements. So, the spatial distribution of base stations is not uniform, since the capacity of connections in an area is basic design criteria for mobile networks, that the capacity of the base stations in any given area at a certain point in time reflects the user mobility, and depends not only on the location of the base stations but also the availability of frequencies that can change over time to follow the users. Since we are interested 
in the variability of the RSSI, in other words, we estimate the level of stability by calculating the RSSI mean.

For instance, from the first measurement, it is seen that the RSSI mean variations of $11.8 \mathrm{dBm}$ for Halotel, $-25.9 \mathrm{dBm}$ for Tigo, $7 \mathrm{dBm}$ for Vodacom and -2.3

Loc. Experiment 1

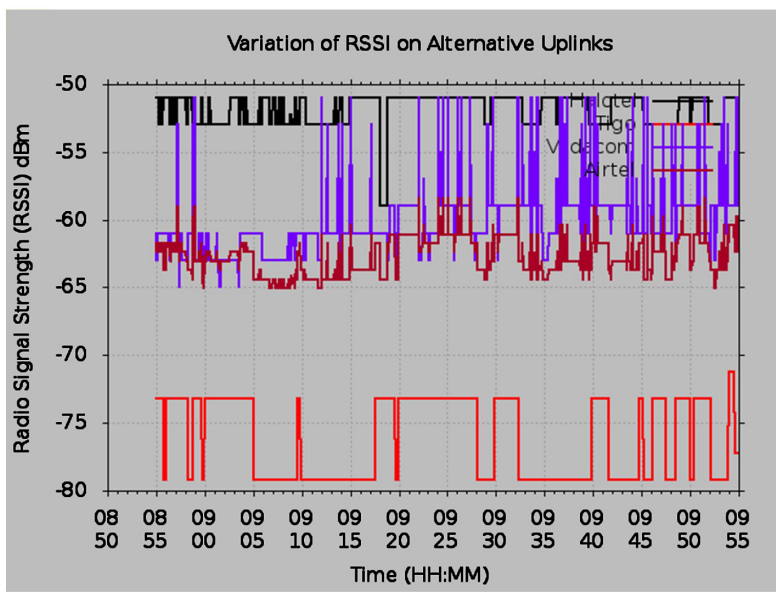

L2
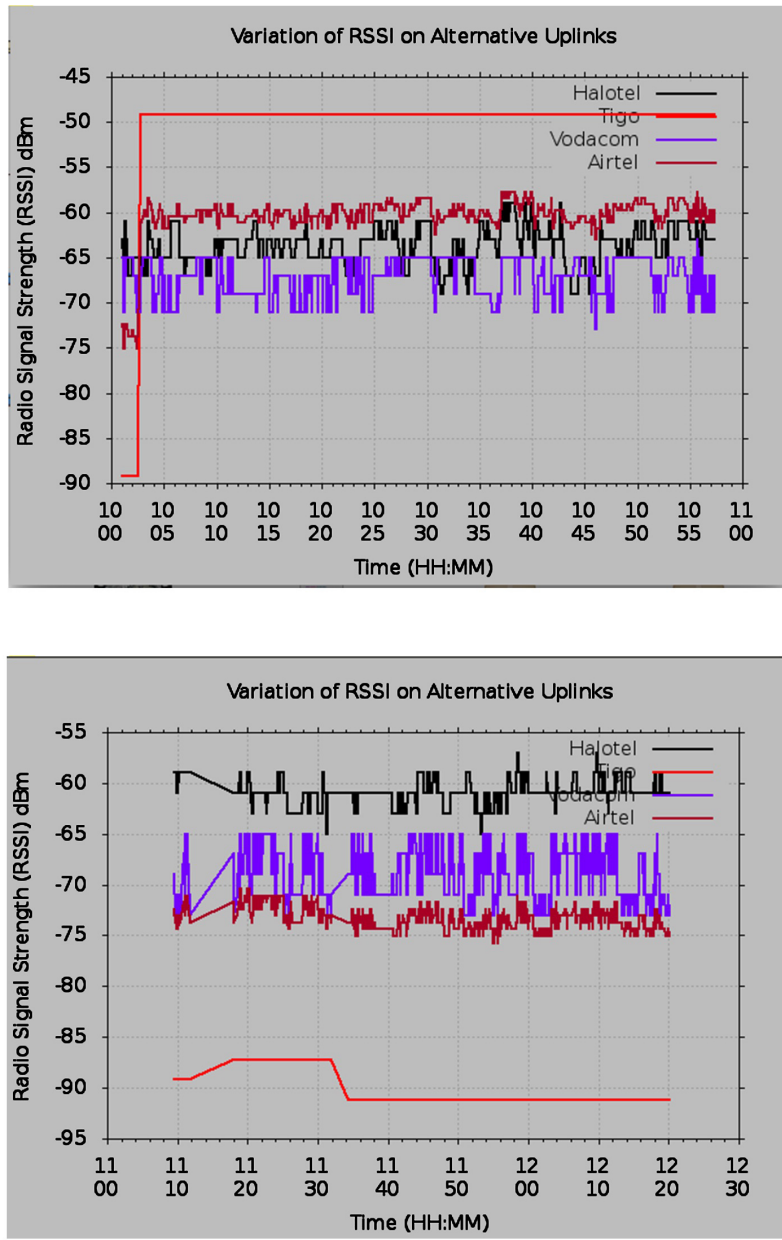

Experiment 2
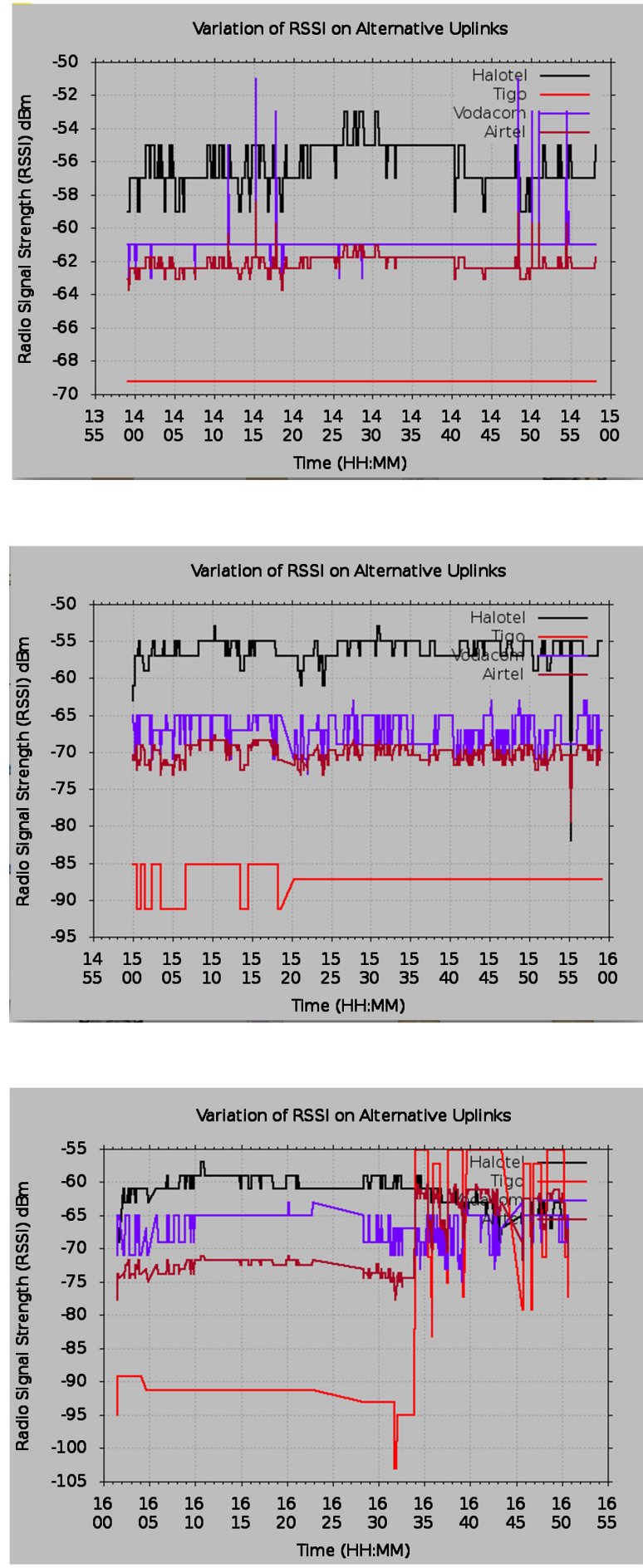

Figure 2. RSSI variations on alternative up-links at locations L1, L2 and L3. 
Table 1. RSSI statistics at location L1 for 4 mobile operators.

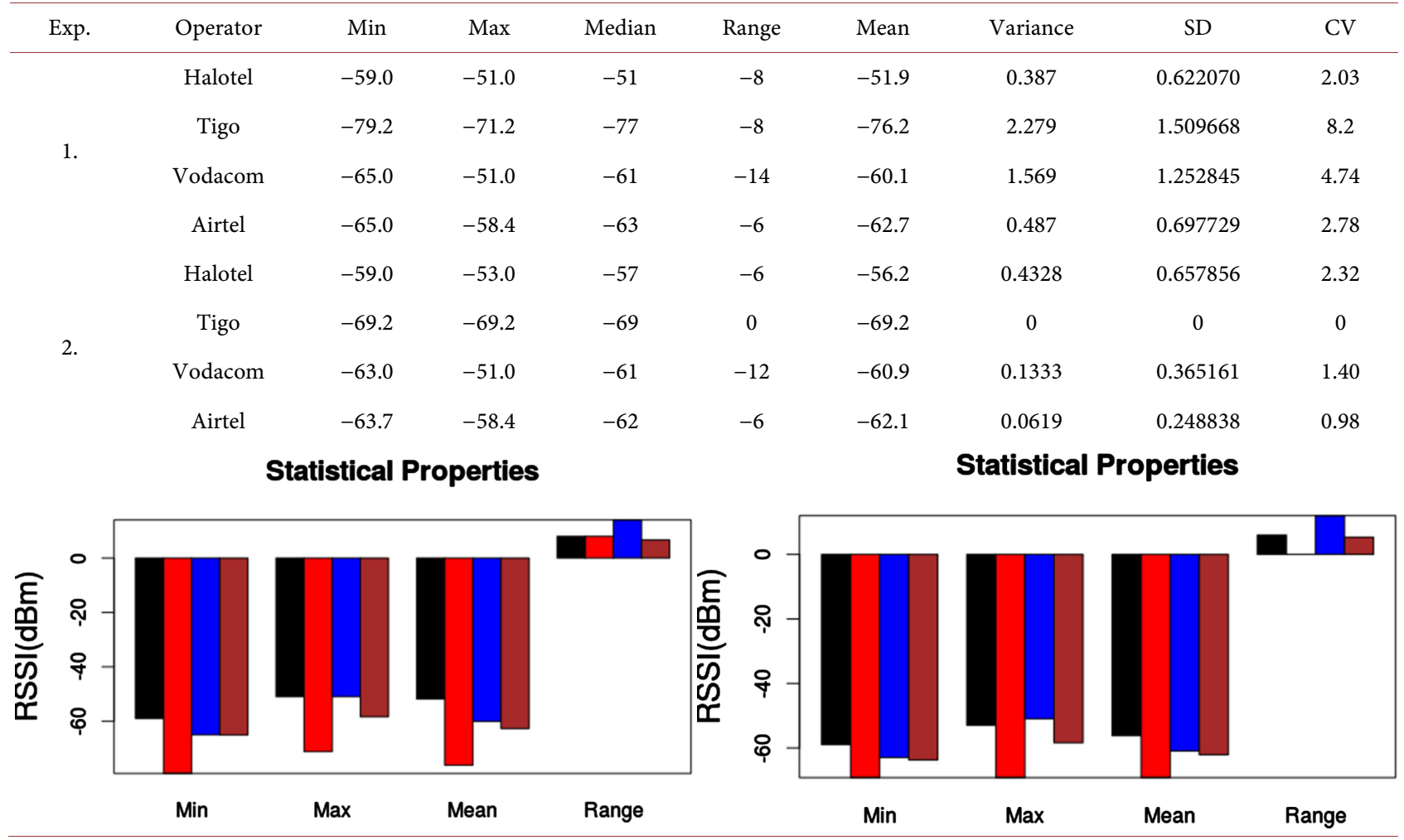

a. Statistical properties of RSSI at location L1.

Table 2. RSSI statistics at location L2 for 4 mobile operators.

\begin{tabular}{ccccccccccc}
\hline Exp. & Operator & Min & Max & Median & Range & Mean & Variance & SD & CV \\
\hline & Halotel & -69.0 & -59.7 & 25 & -10 & -63.7 & 1.1323 & 1.064108 & 4.32 \\
1. & Tigo & -89.2 & -49.2 & 31.9 & -40 & -50.3 & 10.926 & 3.30551 & 10.55 \\
& Vodacom & -73 & -63.1 & 23 & -10 & -67.1 & 1.2189 & 1.104055 & 4.81 \\
& Airtel & -75 & -57.7 & 26.3 & -17.3 & -60.4 & 1.4658 & 1.210702 & 4.60 \\
& Halotel & -82.0 & -53.0 & 29 & -29 & -56.2 & 0.8933 & 0.945119 & 3.33 \\
& Tigo & -91.2 & -85.2 & 12.9 & -6 & -87.2 & 0.6317 & 0.794781 & 6.15 \\
& Vodacom & -73.0 & -63.0 & 23 & -10 & -67.0 & 1.1985 & 1.094746 & 4.76 \\
& Airtel & -79.4 & -67.7 & 21.3 & -11.7 & -70.1 & 0.3559 & 0.596536 & 2.78
\end{tabular}

Statistical Properties

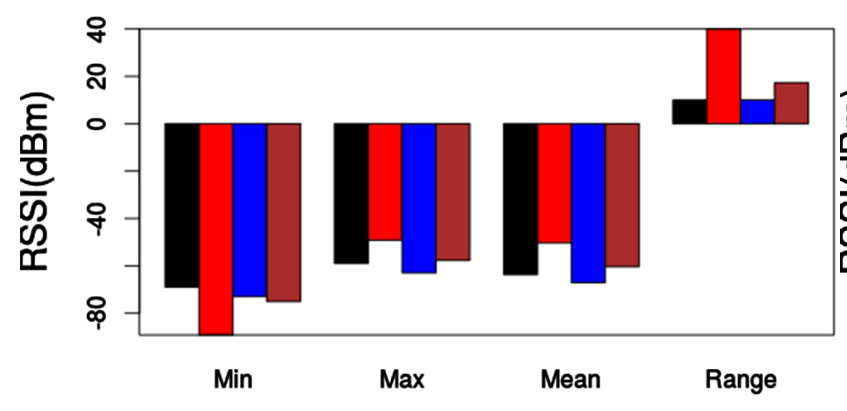

Statistical Properties

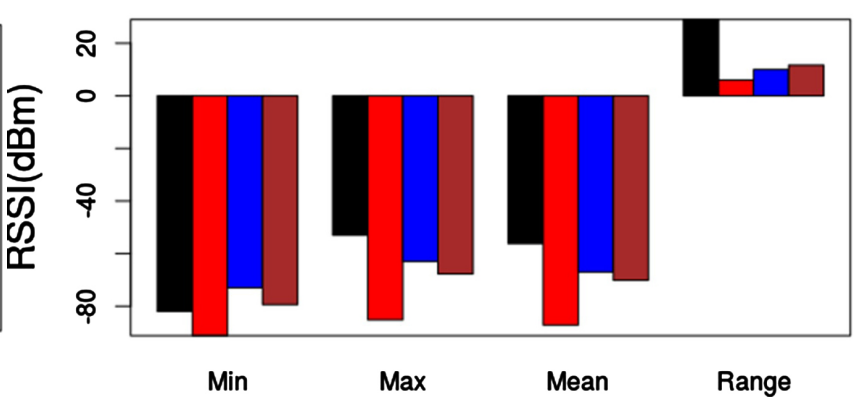

b. Statistical properties of RSSI at location L2. 
Table 3. RSSI statistics at location L2 for 4 mobile operators.

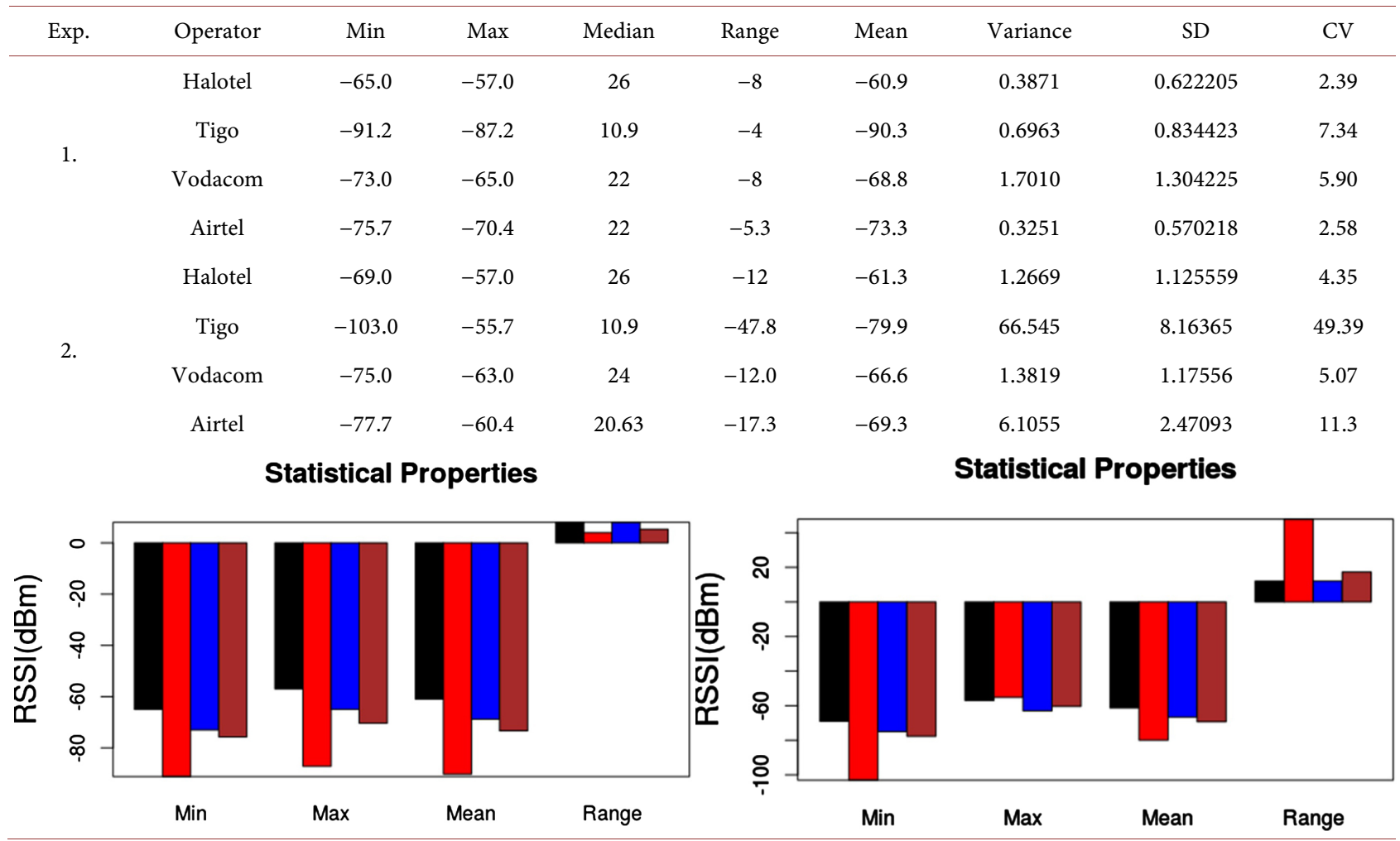

c. Statistical properties of RSSI at location L3.

$\mathrm{dBm}$ for Airtel are observed between locations L1 and L2. Thus, by comparing the results from different operators we conclude that, the location granularity of $3 \mathrm{~m}$ is a considerable difference in terms of RSSI range and mean. The up-link is stable when the RSSI mean is above $-60 \mathrm{dBm}$. But, when the RSSI value is less than $-65 \mathrm{dBm}$, it varies a lot. This means, the up-link is unpredictable if RSSI variability overlaps with a certain RSSI mean.

Temporal variation: We also observe that, the RSSI captured during the daytime may not be the same as the one captured during evening time, due to changes in the environmental characteristics. Several studies confirmed that the temporal variation of RSSI is due to the changes in the environmental characteristics, such as climate conditions (e.g. temperature, humidity), human presence, Interference (e.g. WiFi) and obstacles. Also, the variation of the RSSI may also be due to either constructive or destructive interference in the deployment environment.

Since wireless up-links are highly dynamic, with significant changes in time and surrounding activities. To account for these variations, we measure RSSI from different mobile operators, knowing the amount of standard deviation and measure its coefficient of variation. As shown in Figure 3, the spread or dispersion of the RSSI evaluates how far the up-links deviate from one another. The standard deviation on cellular up-links becomes a kind of average of the differences between the individual up-links and the mean of the up-links at a particular time. From the measurements, we observe that, the variation of RSSI meas- 
ured on Tigo during the morning hours indicates 1.509668 (0.622070 for Halotel) standard deviation close to the mean, with a coefficient of variation of $8 \%$ (2.03\% for Halotel) that spread over short-term variation. Contrary to the measurements measured during noon hours, Tigo indicates 0 ( 0.657856 for Halotel) standard deviation, and coefficient of variation of $0 \%$ (2.32\% for Halotel). Thus, by comparing the results from different up-links we conclude that, a large temporal variation of the RSSI can reach between $(-7 \mathrm{dBm}$ and $4 \mathrm{dBm})$ with a time-scale variation about five (5) hours. The coefficient of variation is high relative to its mean if the rate of variability is high. If there is a low RSSI variation, the coefficient of variation is also low proportion to high level of stability.

\subsection{Up-Link Stability and Robustness}

We analyze the level of stability by considering the robustness of the up-links. Consider the following equation:

$$
\text { Robustness }_{\min }=\text { efficiency }_{\min } / \text { efficiency }_{\max }
$$

The robustness value is normalized between 0 and 1 , and it measures the relative loss of efficiency caused by the poor up-link. If the intermittent link does not impact the efficiency, then its robustness is 1 , while if the intermittent link destroys the efficiency, then the robustness drop to 0 . So, in order to do that, we first compute RTT when the packet size of 236 and 277 bytes are sent to a repository. The RTT quantifies network delay as the duration of time taken by a packet to reach the repository from the gateway plus the duration of time taken by a packet to reach the gateway from the repository. The experiment is repeated at different locations (e.g. L1, L2 and L3), and the RTT mean over all the experiments is obtained as a function of up-link efficiency. It is important to note that RTT measurements are biased by differences in the paths between different modems and the repository they communicate with. The delay between the gateway and the repository decoding it, is mainly a function of the packets travel time, and processing time at the gateway the information traverses.

$$
\text { Latency }=\text { time }_{\text {ack }}+2 * \text { time }_{\text {delay }}
$$

As expressed in the equation above, the RTT or latency is the time for the signal to propagate data or packet from a gateway to a central repository, and send back to a gateway. But the facts expressed by channel efficiency ratio, differ among networks because propagation delay is only one of the various factors affecting the robustness of cellular up-links. Therefore, our goal in this part is to minimize packet losses so that we can obtain the best possible case for robust up-links. An important aspect is to study the link efficiency, which is essential for computing the robustness of cellular networks. Therefore, channel efficiency determines a fraction of the transmission capacity of a particular up-link that contains the amount of data packet to be delivered to a repository. This measures the relative loss of efficiency caused by intermittent links. We expect that the maximum channel utilization can be determined, where data are delivered without transmission errors. 

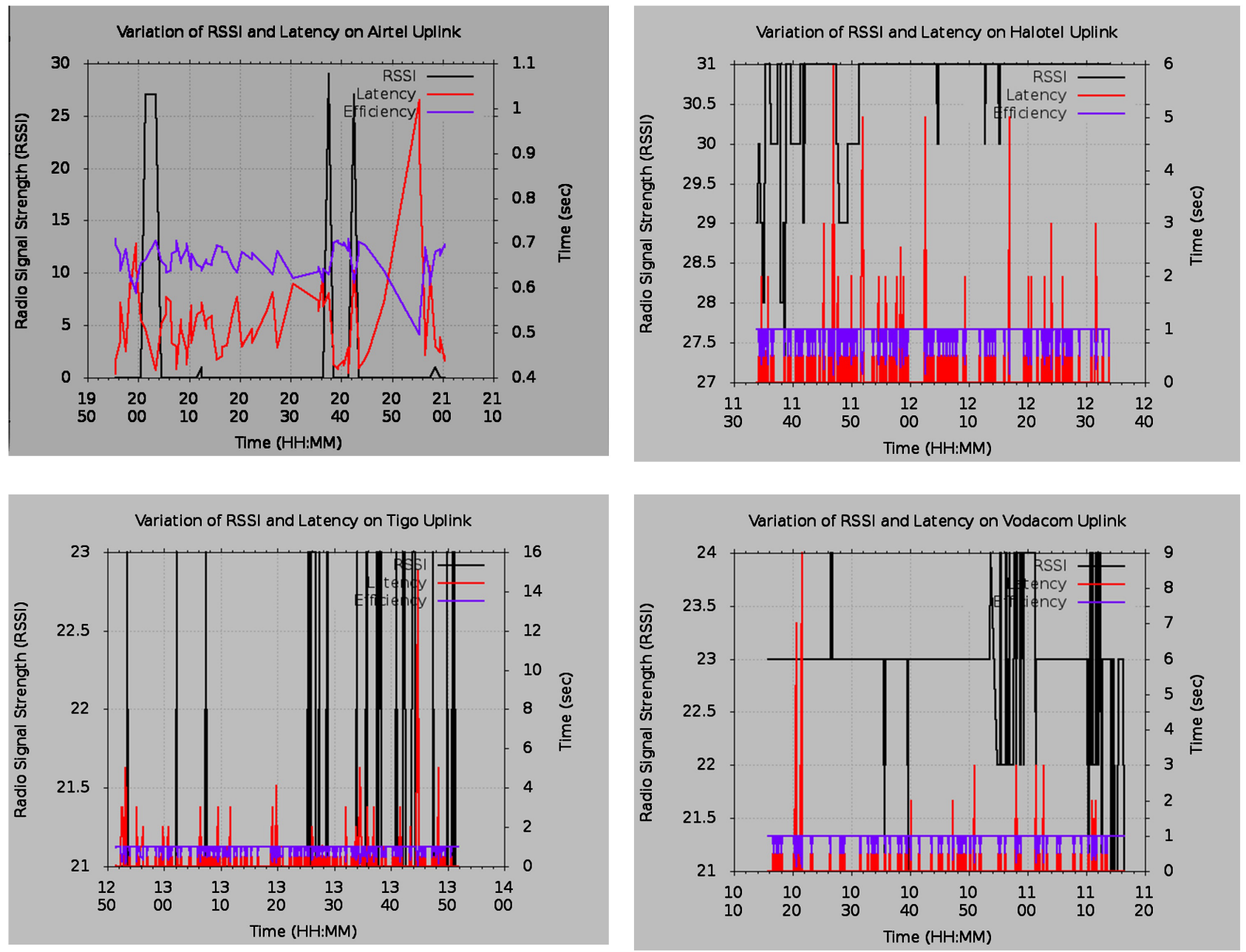

Figure 3. RSSI, latency and efficiency variations on alternative up-links.

$$
\text { channel }_{\text {efficiency }}=\text { time }_{\text {data }} /\left(\text { time }_{\text {data }}+\text { time }_{\text {ack }}+2 * \text { time }_{\text {delay }}\right)
$$

In Figure 3, we try to get a general idea about the stability and robustness of intermittent links, by exploring the relationship among RSSI, latency and channel efficiency. The latency determines that our graphs look like a sinusoidal wave where acknowledgment packet is dynamic. For all intermittent up-links particular when RSSI is small, we see all the high and low-efficiency values. This suggests that, we cannot simply rely on RSSI values as the robustness unit of up-link measure. We need to monitor the throughput of each intermittent up-link to determine the ability to perform as expected under stressful environmental conditions. RSSI and throughput are related in the sense that low RSSI values mean low signal levels which in turn increase transmission errors and therefore retransmits lower throughput.

As shown in Figure 4, we examine time at which throughput varies with RSSI. The variability of RSSI is not proportioned to throughput. This is due to the fact that, the propagation of terrestrial wireless links usually interfering each other, and are further bothered by noises causing packets loss, resulting in reduced 

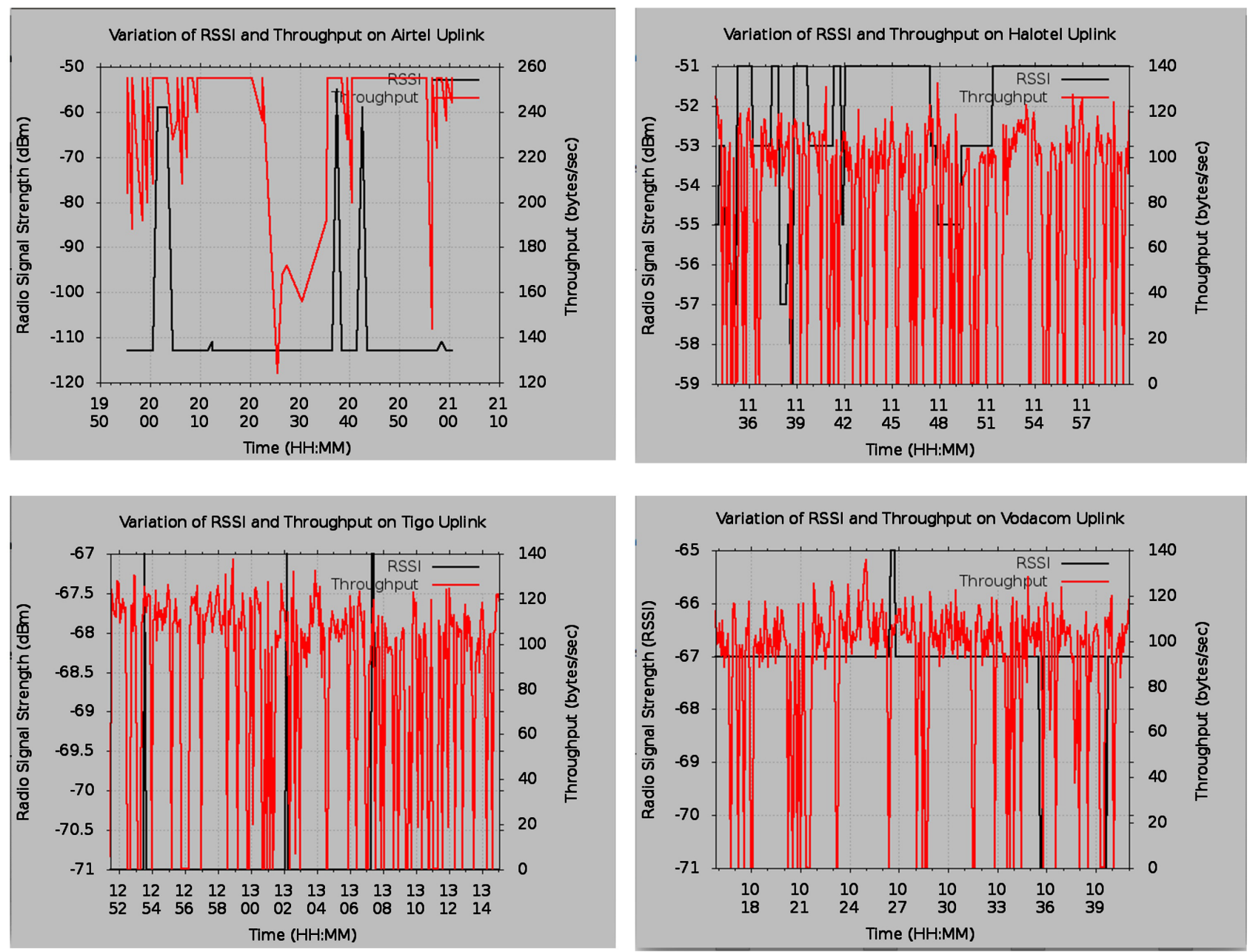

Figure 4. RSSI and throughput variations on alternative up-links.

throughput. Another property of interest is the distribution to which the varying throughput belongs. The distributions at different time are the key properties for studying the robustness of intermittent links. This property is important since we are targeting a time for which throughput can be detected from RSSI. The observations confirmed that the RSSI is not proportion with throughput.

\section{Conclusions and Future Work}

In this paper, we focus on the problem of benchmarking the robustness of cellular up-links based on spatial and temporal effects. We analyze the empirical data from AWS testbed with four (4) cellular up-links on the indoor environment. We show empirically that, the RSSI variations are quite significant. The variations are amplified even further due to some burst transmission, which exists for an unpredictable period of time. To better understand the robustness by considering the level of stability, we consider the following: 1) We present the variability of RSSI based on spatial and temporal effects such as the presence of good reflectors (e.g. metal, walls, woods, and glass) that lead to multi-path (reflections, fading, diffraction) effects, interference, network load or other reasons. 2) We 
present the statistical analysis of RSSI variation, which has distinct values that can discriminate the quality of links. The spatial variation results at least $11 \mathrm{dBm}$ mean deviation on Halotel whereas location granularity of $3 \mathrm{~m}$ leads to up to 11 $\mathrm{dBm}$ mean variation. Further, the temporal variation can reach in between -7 $\mathrm{dBm}$ and $4 \mathrm{dBm}$ with a time-scale variation about five (5) hours. 3) We present the variation of RSSI against latency, efficiency, throughput and evaluate the level of stability by considering the robustness of the up-link against failures.

Thus, we conclude that an up-link with the high level of robustness guarantees a successful packet reception, as the difference between the minimum and maximum efficiency decreases. Our experiments show that Halotel, Vodacom, Airtel and Tigo are robust by $16.7 \%, 10 \%, 7.14 \%, 6.25 \%$ respectively. For many up-links particular when the robustness is small, we can see all the possibilities of the intermittent link. Our future work, intends to extend to explore RSSI threshold, to develop a classification scheme in supporting a decision whether a link is either intermittent or not. This will help in normalizing the level of stability, to design the RSSI estimation metric over intermittent links, for the robust routing protocol in weather data networks.

\section{Acknowledgements}

The authors would like to acknowledge the financial support of NORAD in collaboration with Dares Salaam Institute of Technology for supporting this work under WIMEA-ICT project, with the objective to improve weather information management in East Africa.

\section{References}

[1] Weerasinghe, R.M., et al. (2011) Construction of an Automated Weather Station for Ground Level Weather Measurements. Annual Transactions of IESL, Sri-Lanka, 450-455.

[2] Hut, R.W., van de Giesen, N.C. and Selker, J.S. (2012) The Tahmo Project: Designing an Unconventional Weather Station. EGU General Assembly Conference Abstracts, 14, 8963.

[3] Sharan, R.V. (2014) Development of a Remote Automatic Weather Station with a PC-Based Data Logger. IJHIT, 7, 233-240. https://doi.org/10.14257/ijhit.2014.7.1.19

[4] Tan, C.W., Palomar, D.P. and Chiang, M. (2009) Energy-Robustness Tradeoff in Cellular Network Power Control. IEEE/ACM Transactions on Networking, 17, 912-925. https://doi.org/10.1109/TNET.2008.2003336

[5] Bas, C.U. and Ergen, S.C. (2012) Spatio-Temporal Characteristics of Link Quality in Wireless Sensor Networks. 2012 IEEE Wireless Communications and Networking Conference (WCNC), Shanghai, 1-4 April 2012. https://doi.org/10.1109/WCNC.2012.6213950

[6] Mahimkar, A., et al. (2013) Robust Assessment of Changes in Cellular Networks. Proceedings of the 9th ACM Conference on Emerging Networking Experiments and Technologies, Santa Barbara, California, 9-12 December 2013, 175-186. https://doi.org/10.1145/2535372.2535382

[7] Sebastian, S., Schulte, L. and Manner, J. (2013) Mobile Network Measurements: It's 
Not All about Signal Strength. 2013 IEEE Wireless Communications and Networking Conference (WCNC), Shanghai, 7-10 April 2013.

[8] Md Maruf, A. and Faruque, S. (2016) Propagation Factors Affecting the Performance of 5G Millimeter Wave Radio Channel. 2016 IEEE International Conference on Electro Information Technology (EIT), Grand Forks, ND, 19-21 May 2016.

[9] Thyagaraja, M., Daneshmand, S. and Lachapelle, G. (2016) Assessment of Measurement Distortions in GNSS Antenna Array Space-Time Processing. International Journal of Antennas and Propagation, 2016, Article ID 2154763.

[10] François, Q., et al. (2017) Virtual Multi-Antenna Array for Estimating the Direction of a Transmitter: System, Bounds and Experimental Results. IEEE Transactions on Vehicular Technology, 67, 1510-1520.

[11] Susmitha, P. and Bala, G.S. (2014) Design and Implementation of Weather Monitoring and Controlling System. International Journal of Computer Applications, 97, 19-22.

[12] Kong, D.H., Li, T., You, X.G., Sun, X.M., Wang, B.W. and Liu, Q. (2014) The Research of Long-Distance Data Transmission Based on Meteorological Sensor Network. International Journal of Future Generation Communication and Networking, 7, 59-70. https://doi.org/10.14257/ijfgcn.2014.7.1.06

[13] Jolly, B., Willig, A., McDonald, A., Pannell, M. and Plank, G. (2013) Snowweb-Wirelessly Connected Weather Stations in Antarctica. 2013 IEEE 38 th Conference on Local Computer Networks Workshops (LCN Workshops), Sydney, NSW, 21-24 October 2013, 194-202.

[14] Barrenetxea, G., Ingelrest, F., Schaefer, G., Vetterli, M., Couach, O. and Parlange, M. (2008) Sensorscope: Out-of-The-Box Environmental Monitoring. IEEE International Conference on Information Processing in Sensor Networks IPSN 08, St. Louis, MO, 22-24 April 2008, 332-343. https://doi.org/10.1109/IPSN.2008.28

[15] Le Dinh, T., Hu, W., Sikka, P., Corke, P., Overs, L. and Brosnan, S. (2007) Design and Deployment of a Remote Robust Sensor Network: Experiences from an Outdoor Water Quality Monitoring Network. $32^{\text {nd }}$ IEEE Conference on Local Computer Networks LCN2007, Dublin, 15-18 October 2007, 799-806. https://doi.org/10.1109/LCN.2007.39

[16] Guo, X. and Song, Y. (2010) Design of Automatic Weather Station Based on GSM Module. International Conference on Computer, Mechatronics, Control and Electronic Engineering, Changchun, 24-26 August 2010, Volume 5, 80-82.

[17] Toledano-Ayala, M., Herrera-Ruiz, G., Soto-Zarazúa, G.M., Rivas-Araiza, E.A., Bazán Trujillo, R.D. and Porrás-Trejo, R.E. (2011) Long-Range Wireless Mesh Network for Weather Monitoring in Unfriendly Geographic Conditions. Sensors, 11, 7141-7161.

[18] Eljaam, B. (2005) Customer Satisfaction with Cellular Network Performance: Issues and Analysis.

[19] Scellato, S., et al. (2013) Evaluating Temporal Robustness of Cellular Mobile Networks. IEEE Transaction on Mobile Computing, 12, 105-117. https://doi.org/10.1109/TMC.2011.248

[20] Atmel Corporation. ATmega128RFA1. http://www.atmel.com/devices/ATMEGA128RFA1.aspx

[21] Manges, W.W. (2000) Wireless Sensor Network Topologies. Sensors. http://www.sensorsmag.com/networking-communications/wireless-sensor-network -topologies-778 
[22] IEEE 802.15.4 Available [Online] at IEEE 802.15.4. http://www.ieee802.org/15/pub/TG4.html

[23] Dunkels, A., Österlind, F. and He, Z. (2007) An Adaptive Communication Architecture for Wireless Sensor Networks. Proceedings of the 5 th International Conference on Embedded Networked Sensor Systems, Sydney, 6-9 November 2007, 335-349. http://portal.acm.org/citation.cfm?doid=1322263.1322295

[24] Bhattacharya, A., et al. (2013) SmartConnect: A System for the Design and Deployment of Wireless Sensor Networks. 5 th International Conference on Communication Systems and Networks, Bangalore, 7-10 January 2013, 1-10. https://doi.org/10.1109/COMSNETS.2013.6465582

[25] Srinivasan, K. and Levis, P. (2006) RSSI Is under Appreciated. Proceedings of the 3 rd Workshop on Embedded Networked Sensors, Cambridge, 30-31 May 2006, Vol. 3031, Article ID 239242.

[26] Woo, A., Tong, T. and Culler, D. (2003) Taming the Underlying Challenges of Reliable Multihop Routing in Sensor Networks. In: Proceedings of the 1 st International Conference on Embedded Networked Sensor Systems, ACM, New York, 14-27. https://doi.org/10.1145/958491.958494 